\title{
The Impact of Maternity Service Restrictions Related to Covid-19 on Women's Experiences of Giving Birth in England During the Pandemic: A Qualitative Study and Policy Review
}

Lucy C Irvine ( $\sim$ lucy.irvine@ucl.ac.uk)

Institute for Global Health, University College London

Georgia Chisnall

London Faculty of Public Health and Policy, London School of Hygiene and Tropical Medicine

Cecilia Vindrola-Padros

Department of Targeted Intervention, University College London

\section{Research Article}

Keywords: Maternity services, Covid-19, policy, pregnancy, place of birth, birth partners

Posted Date: February 4th, 2022

DOI: https://doi.org/10.21203/rs.3.rs-1284556/v1

License: (c) (i) This work is licensed under a Creative Commons Attribution 4.0 International License.

Read Full License 


\section{Abstract \\ Background}

The Covid-19 pandemic led to significant changes in maternity service delivery worldwide. In England, service changes included: antenatal appointments being cancelled or held over the phone; women having to attend antenatal scans alone; partners not being allowed to accompany women during labour; visitor restrictions on postnatal wards; and limited postnatal support. On the whole, these changes negatively impacted women's experiences of care.

\section{Methods}

We conducted semi-structured interviews with 46 women aged 18-45 who had low-risk pregnancies and delivered their babies using NHS services in England between 1st March 2020 and 1st March 2021. We also conducted a health policy review to identify guidelines developed for pregnant women and maternity healthcare delivery changes over the duration of the Covid-19 pandemic in the UK.

\section{Results}

Our thematic analysis of interview data generated key themes, four of which are discussed in depth here: the wide reaching negative impact of birth partners not being allowed to accompany women (including impacts on emotional wellbeing, birth preferences and care-seeking choices); women being more concerned about the risk of giving birth alone than of Covid-19 infection; women turning towards private care so that they could have the antenatal care and birth experience they desired; and women feeling deeply frustrated about policy variation between trusts and the inconsistent implementation of guidance.

\section{Conclusions}

Our findings add to the existing literature documenting the negative impacts of service changes on women's birth experiences during emergencies. Some of these impacts could have a positive secondary effect, such as increased uptake of homebirth services. Negative impacts must be addressed by policymakers and health service providers going forward to re-establish trust in NHS maternity care. Existing NHS guidance stating that partners should be recognised as an essential part of a woman's care team should be implemented across all trusts, and any future service restrictions changes should be evidence-based, nationally standardised and clearly communicated to both service providers and users.

\section{Background}

The Covid-19 pandemic led to significant changes in maternity service delivery worldwide that were underwritten by public and institutional-level policies. These primarily involved scaling back normal 
services and restricting face-to-face interactions, including antenatal appointments and limiting the attendance of partners. Early policy changes were made swiftly and without the benefit of the evidence that we now have, nearly two years into the pandemic. While this response was understandable in the face of a poorly-understood disease threat, it led to a return to 'risk averse and intervention-oriented patterns' of care that go against current evidence-informed practice, with detrimental effects on care and the human rights of women and babies ([1] p2). Researchers and policymakers must take this opportunity to learn from these events and make maternity services more resilient for the future.

In response to these unprecedented changes to maternity care provision, various teams of academics have sought to understand the impact on maternity care providers and service users in health systems around the world [1-5]. Given the restrictions on travel and meeting with research participants face to face, much of this research was carried out in online survey formats. This study sought to add to this

body of work by collecting rich data on the impact of Covid-19 on women's experiences of NHS maternity services through semi-structured interviews with a smaller study population. We were particularly interested in how women felt about the way changes to maternity services and policy were communicated and how their preferences for birthplace were affected. A policy analysis was conducted in order to contextualise the experiences reported by service users and to observe how policies played out in practice. We have used these findings to develop recommendations for further research, as well as service and policy improvements.

\section{Covid-19 infection in pregnancy}

When Covid-19 was first identified as a viral disease in January 2020, little was known about its transmission and why certain people suffered from severe illness, whereas others were asymptomatic. In many countries (including England) pregnant women were categorised as a high-risk group by health departments, and advised to follow strict social distancing measures.

Two years into the pandemic, there is more robust evidence available on the clinical outcomes of Covid19 infection for pregnant people and their babies. A scoping review of research conducted in the first nine months of the pandemic found that pregnant women were not at higher risk of Covid-19 infection, but that 'pregnant people with symptomatic Covid-19 may experience more adverse outcomes compared to non-pregnant people' ([6] p10). While the majority of pregnant women who contract Covid-19 experience similar symptoms to non-pregnant women of a similar age, two large retrospective case control studies in the US found that pregnant women were more likely to be admitted to ICU, need mechanical ventilation, and faced a greater risk of death $[7,8]$.

More recently, in England, there has been renewed attention on the clinical risk of Covid-19 for pregnant women because of data released by the NHS which show that unvaccinated pregnant women have made up 20\% of critically ill Covid-19 patients in England between July and October 2021 [9]. This is believed to be, at least in part, a result of inconsistent advice regarding Covid-19 vaccination in pregnancy. Earlier in 2021, pregnant women were advised in government briefings and by health professionals to hold off 
getting the vaccine until there was clearer evidence regarding risk. Anecdotal evidence suggests that misinformation campaigns online may have led some pregnant women to refuse or delay getting the vaccine $[10,11]$. The current best available evidence from both the US V-safe Pregnancy Registry and the UK Health Security Agency vaccine surveillance reporting system suggest that vaccines are safe for pregnant women and do not cause harm to the foetus, with similar rates of stillbirth and low birthweight babies $[12,13]$. In our interviews we asked women if they had been offered a Covid-19 vaccine and how they felt about it. Given the relevance of this topic, we decided to cover it in more depth in a separate paper (forthcoming).

\section{Changes to maternity services in the UK}

As Covid-19 infections spread across the globe in January 2020, NHS leaders and the Department of Health anticipated what policy changes would need to take place to cope with the likely demands on health services. Much of the policy and guidance was based on emerging scientific evidence and the recommendations of SAGE (the Scientific Advisory Group for Emergencies), which provides scientific and technical advice to support government decision makers. While many non-urgent services could be suspended (at least temporarily), maternity services would need to continue to function.

While maternity services met the most essential needs of users throughout the pandemic, certain aspects of care were reduced or suspended entirely. Service modifications in the UK included: maternity staff being redeployed to meet the urgent demand in Covid-19 wards; antenatal appointments being cancelled or being held online or over the phone; women having to attend essential antenatal scans and tests alone; partners not being allowed to accompany women through some or all of labour and delivery (this varied considerably between trusts and over the course of the pandemic, with some not being allowed in at all, others for the period of "active labour" or for c-section delivery only); restrictions on visitors on postnatal wards, limited postnatal care. Jardine et al. [14] surveyed maternity service providers across the UK to investigate local modifications to guidance issued by the Royal College of Obstetricians \& Gynaecologists (RCOG), Royal College of Midwives (RCM) and NHS England. More than two third of the units surveyed reported a reduction in antenatal appointments, and significant changes to specialist care such as women with gestational diabetes mellitus [14]. Over half of units stopped providing some forms of intrapartum care for a week or more, most frequently homebirths [14].

These modifications had a widespread impact on NHS maternity service users throughout their pregnancies and around birth, and on the whole led to poorer quality care than that which was available pre-pandemic. Some of these impacts were relatively minor (for instance, most women did not seem to mind having some of their antenatal appointments via phone), but many had more serious negative effects, such as late diagnosis of treatable conditions in pregnancy. The WRISK-COVID study surveyed 524 NHS service users to explore their experiences of Covid-19 risk messaging and maternity care during the pandemic. The authors found that:

'Communication between NHS organisations and individuals was generally regarded as poor and confusing. Virtual antenatal appointments were fully meeting the needs of only a few users. Restrictions 
placed on partner involvement in maternity care, particularly during scans, emergency attendances, and during labour assessments, caused widespread distress and anxiety...On postnatal wards women described being lonely, sad, and struggling physically without visitors or sufficient staff to support and help them' [15].

Our findings support this and other online surveys carried out in different contexts, and provide rich interview data where we were able to explore the impact of these changes on women's birth experience.

\section{Place of birth preferences}

Choice of birth setting has been a policy priority in the UK for nearly 25 years, with women having the option of delivering in hospital on an obstetric unit (OU) or midwifery led ward (AMU), a freestanding midwifery led unit in the community (FMU), or at home, supported by a homebirth team. A qualitative evidence synthesis found that women's experiences of choosing where to give birth were influenced by information about and preferences for available options, previous birth experiences, and beliefs about risk and safety, among others [16]. Coxon et al. [16] also identified that women found it more difficult to access information and support for homebirth compared to hospital birth. Many of these factors influencing birthplace preference have been affected by the Covid-19 pandemic, particularly beliefs about risk and safety.

Covid-19-related service restrictions influenced women's preferences for where and how they deliver, but these preferences were often not met by service providers. In the UK, news reports suggested an increased interest in maternal request c-section (MRCS) because of women's concerns about whether their partner will be allowed to attend hospital-based vaginal deliveries [17]. Some UK hospitals reportedly introduced a blanket restriction on the provision of MRCS during the peak of the pandemic [18]. Limiting access to MRCS contravenes women's rights to choose where and how they give birth, and compromises their decision-making power.

Despite the anticipated and demonstrable rise in demand for homebirths, around one third of NHS trusts had suspended homebirth services in March 2020 [18]. The justification for this was the increased risk for midwives coming into homes, staff shortages due to Covid-19 infection or needing to self-isolate, and a shortage of ambulances (required for transferring women to hospital if required). These services restrictions varied between trusts and were lifted at different times, adding to confusion and uncertainty among service users.

A smaller number of people considered freebirthing, where someone intentionally gives birth without a health professional present when they would have access to these services. Greenfield et al. [19] explain that out 1700 respondents to their survey on maternity services during lockdown, 72 had seriously considered freebirthing. The reasons given included avoiding hospital settings because of risk of Covid19 infection and concerns around not knowing whether partners could be present during labour in hospital [19]. Many of their respondents had planned for a homebirth, but were considering a freebirth after homebirth services were suspended. 
Similar patterns have been identified in the US, where restrictions to maternity services were introduced in the first year of the pandemic. A quantitative analysis of 3896 women from the Covid-19 Pregnancy Experiences study found that the possibility of contracting Covid-19 in hospital shifted women's perceptions of risk, and led to an increased interest in community birth [20]. Providers in the US interviewed by Gutschow and Davis-Floyd [3] noted an increased number of pregnant people asking for information about homebirth and freestanding birth centres.

Research is ongoing as to whether these changes in birth place preference will continue into the future. Gildner and Thayer [21] hypothesise that the majority of women will continue to opt for hospital birth with physician-led care, a subset of women will pursue out-of-hospital or "community" care in future pregnancies.

\section{Methods}

\section{Study design and research questions}

This study aimed to explore the impact of service changes to NHS maternity services on service users' experiences of pregnancy, birth and the postnatal period. Our objective was to understand what women felt were the most significant effects on their care, how they felt about the way changes to services and policy were communicated and how their preferences for birthplace were affected.

This study involved qualitative data collection alongside a literature review and health policy review. A literature review was conducted by LI in March 2021, and repeated in September 2021 to understand the field and identify relevant ongoing studies. We conducted a health policy review to identify guidelines released for pregnant women and maternity healthcare delivery changes over the duration of the Covid-19 pandemic in the UK in order to contextualise the experiences and perceptions captured by interview data. This followed the framework set out by Tricco et al. [22] for rapid evidence synthesis. GC searched for policies on UK Government, Public Health England (now named the UK Health Security Agency), SAGE, NHS and The Health Foundation's Covid-19 policy tracker databases using the search strategy and inclusion criteria included in Additional File 1. Policies that met the criteria in terms of policy name, date of publication, publishing institution, target population and recommendations made were selected and data extracted. GC summarised the policies using framework analysis [23].

We conducted 46 semi-structured interviews with women via online video and voice calls on MS Teams and Whatsapp, between 25th March and 13th May 2021. 34 of these were by LI, and 12 by GC. Interview length ranged between 30 and 75 minutes. Both interviewers followed a topic guide to ensure interviews covered similar themes, but there was flexibility to allow for women to discuss things that were most important to them.

\section{Recruitment}


Our selection criteria included women aged 18-45 who delivered their babies in between 1st March 2020 and 1st March 2021, using NHS services in England. We recruited women who were considered low-risk during their pregnancy because women who are considered high-risk are usually advised to deliver on the obstetric unit under consultant-led care. While these women ultimately can still choose where they want to deliver, most follow clinical advice, and therefore have a more limited choice of birthplace. Women with high-risk pregnancies are also more likely to experience complications. While their experiences are valid and important to hear, we felt that our team was not qualified to provide the support that would potentially be needed for women when discussing highly traumatic events or poor outcomes.

We recruited through social media channels (Instagram and Facebook) using a research advert. We asked several organisations working in this field to share our post to broaden its reach. Potential participants who contacted us via email and who met the selection criteria were then sent the study information sheet and consent form. Once these were returned, online/phone interviews were arranged at times convenient to the participants. Following a high level of interest from potential participants, and after around 20 interviews, we began to purposively select participants who lived outside of London and who were not White British, in order to record the experiences of a wider demographic. We also selected participants based on their place of birth to ensure we had representation from women who delivered in hospital and at home, as well as by c-section and vaginally.

\section{Data collection and analysis}

Interviews conducted via Whatsapp or Teams were recorded with a separate voice recorder and later transcribed using NVivo transcription software. Key sections were then transcribed manually by LI. All data were stored in a secure online folder in line with the data management protocol. Transcribed data were organised and coded using a rapid assessment procedure sheet (RAP sheet) designed for this study. RAP sheets are used in qualitative research to aide in rapidly identifying common and critical themes in interview data [24]. LI then manually reviewed these and coded the data to identify the key themes discussed below.

\section{Ethical issues}

Discussing pregnancy and birth can be emotionally challenging for many women, especially when this experience has been difficult or traumatic. In our experience, being given the time to reflect on a difficult birth experience in a non-judgemental environment can be useful for some participants. To prepare for women being distressed, LI discussed the topic guide with a representative from Birthrights, an advocacy group, and identified support agencies that we could refer women to in the event that they become distressed in the interview. We were also careful to regularly check in with women about whether they wished to continue the interview. Our views on this were confirmed by many participants noting that it felt like a positive thing to participant in this project and record their experiences, with many hoping it would have some positive impact on services in the future.

\section{Terminology}


All of our participants self-identified as 'women', and so we use this term here, whilst acknowledging that not all people who become pregnant and give birth identify as women.

\section{Results}

\section{Policy review}

Table 1 lists the key national policies and guidelines identified and selected for analysis in the policy review process. These policies and guidelines directly relate to the changes in maternity services in England and were referred to (directly and indirectly) by some participants.

\section{Risk/shielding}

On 8th March 2020, SAGE published a rapid review and expert consensus stating that serious morbidity requiring ICU care occurred in 2/32 pregnant women with Covid-19 (P/G 1). They also stated that $47 \%$ of women hospitalised with Covid-19 were affected by preterm delivery. Despite this, NHS guidance at the time stated that there was 'no evidence that pregnant women who get coronavirus are more at risk of serious complications than the general population or that coronavirus increases the risk of miscarriage' (p. 1, P/G 7), and that women were only encouraged to shield if they had an underlying health condition (P/G 2 \& 7).

\section{Antenatal and postnatal care}

NHS guidance informed women that maternity units may limit visitors to routine antenatal appointments including scans and that partners may not be allowed to ensure social distancing ( $P / G 6$ \& 8). Women were encouraged to speak to their GP/midwife in order to find out more information and that services may be remote, require donning of $P P E$, or be cancelled/re-scheduled (P/G 6). Women were informed that postnatal services and appointments may undergo the same changes $(P / G 8)$.

\section{Labour and birth}

Some of the earliest advice (in April) on the temporary reorganisation of services acknowledged that trusts had reported imposing restrictions on visitors, but stated that 'women should have access to one birth partner during labour (from the point of admission to labour ward or birth centre)' (p. 3, P/G 3). Later NHS guidance informed women that there may be changes to where they plan to give birth and that they should speak with their maternity team for more information (P/G 6 \& 8) and look for relevant websites or social media sites ( $P / G$ 8). Guidance also specified that while women are entitled to a birthing partner during labour and birth that there may be limits on how long they will be able to stay after birth (P/G 6).

Further NHS guidance for women re-enforced that birth experience still mattered during the Covid-19 pandemic (p. 1, P/G 8):

'You can expect: 
- to be treated with dignity and respect

- to have a birth partner of your choice

- to have access to pain relief options

- to make informed decisions about your birth

- to have clear communication from maternity staff

- to plan to give birth in a setting that is right for you

- to be mobile where possible and give birth in the position of your choice'

A caveat was included, stating that some changes may be necessary in order to maintain safety, including suspending or reducing homebirth or midwife led services.

Healthcare provider guidance urged that services should maximise choices for women within the constraints of staffing and facilities (P/G 3). This healthcare provider guidance outlined that even in instances of restricting visitors for infection control, women should have access to a birth partner during labour, this being important for maternal wellbeing and in providing additional practical support. This guidance also urged healthcare providers to understand the impacts and potential risks of women's mental health during this time due to the pandemic and changes in service provision.

\section{Reintegration of partners into maternity care}

On 14th December 2020, NHS England published new guidance (P/G 5) to replace the guidance published 8th September 2020 (P/G 4) regarding the re-introduction of partners in maternity services. The September guidance recommended a stepwise approach to reintroducing partners to maternity services and supported local context response. The updated guidance stated that all trusts should 'urgently complete any further action needed so that partners can accompany women to all appointments and throughout birth' (p. 1) and emphasised that women should 'have access to support at all times during their maternity journey and trusts should facilitate this' (p. 2) (P/G 5). Furthermore, the guidance outlined that partners should not be considered 'visitors' but core components of the women's and baby's care ( $\mathrm{p}$. 2) and hence be present at all scans, appointments and labour/birth from the point of hospital attendance. The guidance states that all families and relevant staff should receive clear communication on this update.

\section{Participant characteristics}

All 46 women participating in our study delivered their babies between 1st March 2020 and 1st March 2021, using NHS maternity services in England. For most participants (33), this was their first baby. In general, women who had had another child before the pandemic said that they were better equipped to cope with the challenges of having a baby during it.

Participants ages ranged from 28 to 44, with the mean age 33. Around half of women interviewed (24) lived and accessed their care in London, with the other half widely spread across England. We used job 
title as a proxy for approximate income level, and from this deduced that most participants were within the lower-middle to upper-middle income brackets for England. The majority of participants $(80 \%)$ described themselves as White British. 9 women self-identified as Black and White African, Indian, White European, White Jewish, or other/mixed. More detailed participant characteristics are available in Additional File 1.

Table 2 shows that nearly $20 \%$ of participants opted for a homebirth, with $13 \%$ actually delivering at home. This is significantly higher than the percentage of women who normally give birth at home in England and Wales, which was $1.9 \%$ in 2019 and has remained fairly consistent over the past decade [25]. This is likely to be a result of our selective sampling for a broad representation of birthplace settings and modes during recruitment, and the dissemination of the study advert on birth-related social media groups, which tend to promote messaging on normalising birth and the benefits of homebirth and midwifery-led care. However, it is also possible that the higher-than-normal interest in homebirth is at least in part a result of the pandemic and the restrictions on normal maternity services. This is reflected in our interview data below, as well as ONS data that recorded a slight rise in homebirths in 2020 compared with 2019, and the lowest level of NHS hospital-based births in the past decade (96.4\%) [25].

\section{Interview results}

Our policy review outlines the relevant policies and guidance from the UK government, NHS England, SAGE and RCOG. While the earliest advice suggested women should shield, from then onwards this guidance stressed the importance of upholding high-quality women-centred care and facilitating a positive birth experience amidst the pandemic. This specifically included being treated with dignity and respect, having a birth partner of your choice, and receiving clear communication from maternity staff. Our findings highlight serious failures to provide this.

\section{The key role of partners}

From the early onset of the pandemic, women's partners (most often their spouse, but also family members, friends or doulas) were restricted from: attending antenatal appointments and scans; being in hospital with women during the early stages of labour and induction; and staying on the postnatal ward for more than a short number of hours. While all of the partners of the women we interviewed were allowed to be present for some part of the birth, many mentioned friends who had to go through the entire labour alone.

UK government guidance from April (P/G 3) stated that partners should be allowed to accompany women from admission onwards, but was open to interpretation by trusts based upon context-specific discretion. This resulted in a "postcode lottery" of care, where some women could be accompanied by their partner throughout their entire labour and the postnatal period, whereas other women's partners were refused entry during early labour, or were asked to leave shortly after birth. Almost all of our participants reported the negative impact this had on their experiences of pregnancy and birth. While a small number of women felt they could manage (especially if they already had children and so felt more confident about labour and how to care for an infant) others were extremely distressed and some went on to have serious 
postnatal mental health issues. They attributed these, in part, to being left to cope alone with a new and challenging situation that they had not anticipated.

For women who were having their first baby, or for those who had previously had miscarriages, going to antenatal scans alone was particularly worrying, with many reporting feelings of extreme anxiety.

For me, that was very stressful, because obviously l'd had three miscarriages before and we'd found out through scans for two of them. So to go on my own, to sit in the waiting room was pretty horrific Women also mentioned the negative impacts of these restrictions on their partners, explaining that they felt distant or even excluded from the pregnancy.

For the 12 week scan I went on my own, my partner waited outside the hospital for me, and it was really hard, because you know, there's a lot happening, it's your first scan, and I was there on my own. And I felt just really sad and disappointed

Other participants described how their partners had found it harder to bond with their babies as a result of not attending appointments. At the same time, this left some women feeling like they had taken on an even greater burden of responsibility for the pregnancy and the baby.

The lack of partner support during delivery and in the days following was a common experience among our participants. Several women described how surprised they were when their husbands were asked to leave immediately after birth, that they weren't prepared to say goodbye, and that they had barely had time to acknowledge or celebrate the birth of their child.

"Then my husband was kicked out... I had no time to consider what had just happened... He was just told to walk the other way down the corridor, we hadn't even looked at each other to say 'well done on having this baby"'

He wasn't even allowed to go with me to take my things in, to help me settle into this new space, to say goodbye...And he almost cried [gets upset and cries]. It was really hard, me being separated from my partner, after everything we had been through. We didn't even have a chance to decompress, to process what happened

There was evidently a lack of communication around the restrictions on postnatal wards that took women by surprise, and affected them more acutely as a result. Participants also commented on how difficult their stay on the postnatal ward was, primarily because they had no emotional or physical support from their partner.

Those five days [on the postnatal ward] make me more emotional and feel more traumatised than the labour did...I was in a lot of pain and then our baby was sick... Then to be on my own, all alone with the baby for five days - I don't think I slept for three or four of the days, between trying to feed him and look after him and being anxious. It felt like some sort of torture. It felt like I was locked in there and I couldn't leave 
"It was the middle of the night, I couldn't sit up out of the bed - because I had no core strength and I had all these stitches - to attend to the baby, so I started feeling quite nervous to be left alone, after going through this crazy life experience. And all of the emotions, and everything that goes along with that, being left with an infant, when I could hardly even move"

Overall, women felt extremely frustrated that they were not able to have the consistent presence and support of their partner throughout the entire pregnancy and perinatal period, as they normally would have if they chose. While women were understanding of the emergent situation, and the decisions made by trust management to err on the side of caution when so much was still unknown about Covid-19 in the early days of the pandemic, over the time period studied, frustrations developed.

Why are we still not able to have the support that we need? Why are birthing partners and dads being treated like visitors, when they're not - they're a vital part of the maternity team?

Women felt that their partners were seen as non-essential to a positive birth experience, whereas in fact, for most women they are a core component of their care team, especially in labour and during the immediate postnatal period.

\section{Weighing up risk}

We asked women how they felt about the risk of Covid-19 itself during their pregnancies and afterwards. Interestingly, although they were not asked to make any comparison, many of the women we interviewed made it explicitly clear that they were more concerned about having to labour and give birth without the support of their partner than they were about the risk of contracting Covid-19. Some participants did express concern about the risk Covid-19 itself posed to them and their babies, especially earlier on in the pandemic period when pregnant women were advised to shield along with highly vulnerable groups as a precaution. The majority of women interviewed followed this government advice on social distancing and/or shielding throughout their pregnancies, limiting their risk of infection. Despite this, for almost all of our participants, the indirect risk Covid-19 posed, through the impact on services, was of far greater concern. This anxiety was almost entirely related to having to be without their partner for some stage of the birth. This was most pronounced among women who delivered a month or more into the period studied. By this point, stories about having to deliver alone were circulating in the news and on social media groups related to birth. When women sought clarity from their midwives or their trust maternity helpline, they could normally not guarantee that women would be accompanied throughout labour, due to the policies in place at the time (which were normally that partners could only join women once they were in "established labour", when their cervix has dilated to $4 \mathrm{~cm}$ ).

I was a little concerned about Covid, especially when we didn't know too much early on...But I was more worried about my husband not being able to be with me than I was about contracting the virus

... I probably should have been more scared [about Covid-19]. But I wasn't. I knew we were being careful... my biggest concern about the whole thing, was having to have a c-section because of Covid, then having to stay in hospital for longer without support and without my partner

Page $12 / 23$ 
Some women were so concerned about this risk that they made decisions about their care to make sure their partner could be there for as much of the birth as possible. This included labouring at home for as long as possible before going into hospital, and opting for a homebirth.

Covid pushed me to make that decision [to get a homebirth]... We were very careful with shielding a few weeks before my due date, just because I was scared not to have my husband there. So I was more worried about the restrictions due to Covid in hospital, as opposed to actual Covid

"... we still wanted a homebirth. Covid sort of consolidated our desire for it really because we didn't want to be anywhere near a hospital"

I can't really describe how grateful we felt that we had already made the decision that we had, not only to have a homebirth, but also to go with independent midwives...I wouldn't have been allowed one under the NHS care...As we learned about all the restrictions that would have been placed on us, my husband not being able to be with me afterwards or even in active labour - whereas in the homebirth, he never left my side. I couldn't imagine it being any other way... I feel hugely emotional about the fact that so many women during this time were denied that

Some participants who had had negative experiences in hospital explained that they would consider alternative options for future pregnancies. Most stated they would seriously consider a homebirth if they had other children, in part because they thought they would have a more positive birth experience. Participants attributed negative outcomes (increased pain and tearing of the perinium) to travelling into hospital mid-labour and the clinical environment of the obstetric unit. But all of these women also mentioned that having their partners present for the entire labour and postnatal period were key factors in their decision making.

Other women made the decision to leave the postnatal ward sooner than they might have normally, so that they could be at home with the support of their partners. One participant explained how she struggled to manage with her newborn alone on the post-natal ward after an emergency c-section, and so left the following day:

Throughout the night...everything was really, really difficult. We hardly slept, it was really noisy, and I felt absolutely horrendous. I said to them that I couldn't cope in there on my own, I needed someone with me, and that I wanted to go home that day. They said that was fine, so long as I could make it to the bathroom. I was surprised, as I thought normally after a c-section you had to stay in for two days, but they were quite happy to let me go. I dragged myself to the toilet, almost on my hands and knees... because I just couldn't stand another night there on my own.... if it wasn't for Covid, I would have stayed in longer, and had more support with breast feeding [and access to stronger pain relief].

These interview excerpts demonstrate how women were deliberately choosing to delay going to hospital, avoid it completely, or leave earlier than they would have otherwise, not because of the risk of Covid-19 infection, but because of the restrictions on partners attending. In some cases, this meant that women were at higher risks of complications, but that they saw this as a risk they were willing to take, compared with struggling alone in hospital. 


\section{Use of private health services to fill Covid-related gaps in care}

Some participants explained that their negative experiences had led them to opt for private care to fill gaps in services during the pandemic, or to consider private care during a subsequent pregnancy. This was usually by paying for additional private ultrasound scans so that their partners could attend (which was permitted throughout the pandemic in most private clinics). A small number of women contracted a private midwife, usually because they had decided on a homebirth, and NHS homebirth teams were suspended for specific periods by some trusts during the pandemic. Others expressed a wish that they had, or explained that they would pursue this option in future.

I personally wish that I'd had an independent midwife, I feel like that would have made a difference... people in [my Whatsapp baby group] booked their own private scans, because they felt like things were being missed, or not discussed properly...I've already told one of my friends to put some money in her budget for postnatal support because it's not great [on the NHS]

Well, we do want to have a second baby and we've had, I guess, some casual conversations about whether we should we go private for the second one. I have a friend who is going private at the Portland Women's Hospital because she decided she makes enough money to afford it and she decided that it's just too stressful with the NHS

To be honest, I've completely given up with the NHS, and I've been completely spoilt with a private midwife and I wouldn't bother with a doula again, I would just have a private midwife because they're worth every penny. Which is awful because I know not everyone can afford it, but I just think if you can, just do it. Because the NHS services can't be trusted

The way that women describe their decision making process here suggests women are seeking out private midwives not because they have a particularly strong vision of what their birth experience should be (some women choose independent midwives because they tend to have a strongly naturalistic view of birth) but because NHS services were not meeting what they saw as a reasonable standard of care. The inconsistency (suspending then reintroducing homebirth services) and the changing rules regarding partners were two key issues that women sought to circumnavigate by paying for private services.

One participant went into detail about her traumatic birth experience. She felt that she was induced unnecessarily, so that her labour was faster and thus more convenient for the already strained services during the pandemic. As a result, she had to labour on the antenatal ward for two days alone.

I think if I was to have another baby, I would definitely stay at home for as long as possible. I'd even consider a homebirth, and I've never considered a homebirth before because I wanted I wanted the epidural...[After the birth] I was having horrific nightmares about my labour, 2 or 3 times a week, waking up with heart palpitations, sweating. I've got to take the baby for a hospital check-up this month, and I've got anxiety... I just never want to go into a hospital ever again...I would consider giving birth at home because I know now that my body can do it naturally, and it wouldn't be worth it to me, to go back into one of those places. I don't trust them anymore, I don't trust midwives, I don't trust the doctors. I felt like I 
was bullied and manipulated into doing things that as a first-time mum with no experience and no support system, into doing things that suited them, and the way they were running things during the pandemic

This experience left her with anxiety about returning to any kind of clinical setting, and with a lack of trust of medical professionals. This kind of outcome is clearly undesirable for all involved in providing maternity care.

The women who did opt for private care were aware of the fact that paying for maternity care was fairly unusual in the UK, and that they were in a privileged position to be able to afford a private independent midwife (the cost of which is typically between $£ 2000-£ 5000$ ). What is particularly concerning here is that women felt driven to pay out of pocket because they were unable to access services that should normally be delivered on the NHS and usually are accessible to all, free at the point of use (namely, scans with their partner present, and homebirths). Many women could not afford to do this, and these kinds of financial barriers drive inequalities in access to essential maternity services.

\section{Policy implementation and government priorities}

Our participants were particularly frustrated about the inconsistencies in policy implementation between trusts, and the fact that restrictions on partners were still in place when other lockdown measures were easing.

"When the new guidelines [on partners] got released, everyone was so excited - the community online, the people that had campaigned for it...but obviously every hospital got to make their own decisions and even...you just didn't know what you were going to get"

It was ridiculous. There was a trust in South London that had no partners until the very end of birth, some were having in person appointments, some were having phone appointments. In my NCT group there were 3 different hospitals that we were going to. And they were all communicating things differently There was a widespread disbelief among our interviewees that in the Autumn of 2020, their partners could still not come into hospital to support them in labour, but that hospitality venues - particularly pubs - were opening in the UK, which seemed to encapsulate for them how misaligned the government's priorities were with their needs.

Partners should be able to come to appointments, partners should be able to be there for the whole delivery, partners should be there for postnatal. We need that support, and the midwives, they can't give that same level of care to us than our partners could. The thing that made me really sad was that we were being denied that, while it was still ok for people to go to restaurants and pubs...but we weren't allowed to be one to one with the person who is most important to us at that moment

I was contacting our MP to push for them to contact the hospital to push for changing of restrictions... especially when it was October and we could go to the pub. I kind of joked about having the baby at the pub so my husband could be there 
Several women had actively campaigned at the local level for more consistent policy implementation. One had written a formal appeal to the Whittington to allow her partner or independent midwife to accompany her in the event of a transfer during homebirth, based on the guidelines from RCOG at the time that recommended upholding continuity of care. Her request was declined and she was told that the trust was allowed to make its own interpretation of the guidance. She and other participants felt that the reasoning behind these decisions was unclear and even irrational. Many participants felt that their wellbeing was not being considered as a priority - which suggests a failure to provide high-quality respectful maternity care throughout the pandemic, despite this being a policy priority in messaging and guidance from central NHS England, RCOG, and other key actors in maternity care provision.

\section{Discussion}

Our findings are consistent with survey research conducted in the UK with NHS service users, as well as other qualitative research carried out in contexts such as the US where similar service restrictions were implemented [2-5]. By conducting in-depth interviews with participants, we were able to investigate women's concerns further and explore certain topics in more depth, which we have reported here.

Partners play a fundamentally important role in supporting women through pregnancy, labour, and the postnatal period. The benefits of continuous emotional support during birth are well established in research, and are set out in a Cochrane systematic review of the literature [26]. In England, the majority women are usually accompanied by their partner [27] and in some cases a doula, friend or family member. During the pandemic, many women had to cope with some of the challenges of pregnancy, labour, and caring for a newborn alone, which for some, had serious impacts on their mental health and emotional wellbeing. This study contributes to a growing body of evidence that the Covid-19 pandemic has exacerbated feeling of fear, anxiety, stress and a loss of agency that women can experience during pregnancy [28]. Of particular importance was the inconsistency and abruptness in which partner restrictions were communicated to women, such as when women were waiting up until the last minute to find out if their partners could attend antenatal scans, and when they were given no time to process the birth of their children with their partner before being moved onto the postnatal ward.

The importance of partner support was further reflected in the comparisons women drew between the risk of contracting Covid-19 and the risk of giving birth without their partners in attendance. While some of our participants were concerned about infection, especially earlier on in the time period studied when the government advice was for women to shield, almost all saw this as a lower concern than having labouring alone. To our knowledge, women explicitly describing this decision-making process as a weighing up of comparable risks is a unique finding of this study. This has significant implications for understanding women's birth preferences during the ongoing pandemic, and may aide policymakers in their efforts to ensure the provision of women centred care.

Several participants cited the risk of partners not being allowed as one of the key decision-making factors that had led them to seek out alternative options for care. For some, this meant choosing to delay going 
to hospital for as long as possible, or to deliver at home when they might not have previously considered this option. Nearly $20 \%$ of our participants planned for a homebirth, with $13 \%$ actually delivering at home - a significantly higher rate than the 2019 national average of $2.1 \%$ [25]. The rise in interest in out-ofhospital birth is reflected in studies carried out in the US. In a national survey of women who delivered during the pandemic, there was a greater than $200 \%$ increase in the number of participants who reported a novel preference for community care following the pandemic, compared to those who had already preferred community care [21]. This greater demand for out-of-hospital births was not met by NHS providers during the periods when homebirth teams were suspended.

A sustained increased interest in homebirth could have potential benefits for women and their babies, both in terms of clinical outcomes and in the likelihood of having a positive birth experience. The comprehensive prospective cohort study carried out by the Birthplace in England Collaborative Group [29] found that for multiparous women planning birth at home experience fewer interventions and negative maternal outcomes that those planning birth on an obstetric unit, with no impact on perinatal outcomes (though for nulliparous women, there is some evidence that planning a home birth is associated with a slightly higher risk of poor perinatal outcomes than in an OU). These findings are upheld by a more recent systematic review of maternal outcomes in different birth settings [30]. Smaller scale qualitative studies conducted in Israel [31], Finland [32] and Sweden [33] have found that homebirth is generally associated with a more positive subjective birth experience, as described by women and when accounting for their pre-existing views on childbirth. Whether or not this increased demand for homebirths continues remains to be seen, but it could have a long-term positive impact, giving women greater choice and more confidence in out-of-hospital birthplace services. Other authors also express optimism about this shift in the US context, suggesting that it could be a 'transformational moment' that could move maternity care 'toward a more integrated and sustainable model that might improve provider and maternal experiences as well as maternal and newborn outcomes' ([3] p1).

Another finding which we believe to be unique to this study is that Covid-19 restrictions were a factor in women deciding to pay for private scans or private midwives. In the case of the latter, this was a considerable expense for the women we spoke to, but one that they thought was worthwhile. This is a particularly interesting in the UK context because of the cultural importance of the NHS, and the value that health professionals and service users ascribe to being able to access high-quality care free at the point of use [34]. The fact that women are considering paying out-of-pocket for private care because they cannot attain quality women-centred care through the NHS should be deeply troubling to health policy makers, providers, and government. This has the potential to widen existing health inequalities between different population groups, and counters the long-term efforts of those in the health sector working to ensure equity of access to high-quality, woman-centred care.

\section{Recommendations}

Further research could build on the findings of this study. The ongoing impact of Covid-19 on maternity services is important to understand, particularly: if there are any long-lasting mental health problems for

Page $17 / 23$ 
mothers, and if the shift in birthplace preferences is sustained or returns to pre-pandemic patterns. Posing similar research questions in other country health systems could lead to useful comparisons and potential lesson-learning, particularly those that managed to minimise the impact on maternity services.

\section{Partners}

In December 2020, official guidance from NHS England stated that women should be permitted to have one person accompany them at all stages of their maternity [35]. As our findings suggest, this guidance was not universally implemented across NHS trusts. A 2021 update of this guidance goes further in asking all trusts to implement this guidance, undertaking risk assessments, reconfiguring space and using available testing capacity to achieve this [35]. Going forward, and given the current high rates of Covid-19 cases resulting from the Omicron variant, facilitating partner access throughout pregnancy, birth, and the postnatal period should be of paramount importance. In December 2021 we observed social media notifications from some trusts advising women that due to the high case numbers, only one birth partner is currently permitted, rather than two. Our data highlight the importance of maintaining a minimum of one partner throughout labour and the postnatal period.

\section{Policy consistency and communication}

Our findings confirm that one of the main frustrations experienced by women was the inconsistent application of policy guidance across the country, with wide variation even between neighbouring trusts. Some trusts communicated policy changes efficiently, whereas in other cases women were left to find out information on their own, and were sometimes not informed of changes that impacted upon their care until the last minute. While some of this was to be expected in the early days of the pandemic response, nearly two years in there should be greater consistency and communication. Having a national, unified, evidence-based policy approach to any restrictions to services would be a positive step.

Lessons might also be identified from other country health systems that enacted distinct policy measures. Taiwan implemented tighter infection control measures nationally, and these included for clinical settings. One maternity hospital team established a specialised ward for women with Covid-19 (with separate entrances), had strict temperature testing and limited site entrances where people had to complete health declaration forms [36]. This meant that women could continue to have up to two visitors with her, one of which could stay overnight. A more comprehensive and universally implemented infection control system could protect maternity staff whilst ensuring women can be accompanied by one, or even two birth partners throughout.

\section{Study limitations and strengths}

In recruitment, we aimed to interview a group of women from diverse sociocultural and ethnic backgrounds in order to capture a range of experiences, and also to observe any potential differences in how they felt about their birth experience, including any differential treatment. We actively recruited through channels that would reach minority communities, such as Instagram accounts of organisations working with these groups. Despite these efforts, there was an overrepresentation of women who 
identified as 'White British'. Women who identified as being from Black, Asian or mixed ethnicities did not report any discrimination, though because both interviewers are white, they may not have felt comfortable discussing it. This was one of the limitations of the study. It would be important to understand whether women from minority ethnic communities' care was even more adversely affected during the pandemic, especially given that they are at higher risk of perinatal mortality and morbidity compared to their white counterparts.

Another limitation was that due to social distancing guidance in place at the time, interviews were conducted on the phone or online rather than face to face. Despite this, we did not find women to be hesitant about communicating online. By this point, a year into the pandemic, many people were used to video calls. This method was also more convenient for women with babies than meeting in person, and meant they could conduct the interview while at home.

A strength of this study is the rich data collected through in-depth semi-structured interviews. We were able to spend time listening to women's concerns, and to ask for clarification about certain points, which is an advantage over survey data collection.

\section{Conclusion}

Our findings add to the existing literature documenting the negative impact of service changes on women's birth experiences. Some of these impacts could have a positive secondary effect, such as increased interest and uptake of homebirth services. Nonetheless, negative impacts must be addressed by policymakers and health service providers going forward to re-establish trust in NHS maternity care. Our recommendations are that birth partners should always be recognised as an essential part of a woman's care team, with a minimum of one partner being permitted to accompany women at all times throughout labour and the post-natal period (especially now that the risks of severe disease from Covid19 infection have been greatly reduced through vaccination campaigns). Furthermore, any future service changes should be standardised across all trusts and clearly communicated.

\section{Declarations}

\section{Ethics approval and consent to participate}

This study was conducted in accordance with the Declaration of Helsinki and informed consent has been obtained from all participants included in this study. This study was granted ethical approval by the University College London Research Ethics Committee (Project ID: 19863/001).

\section{Consent for publication}

Not applicable.

\section{Availability of data and materials}


Due to prior agreement with research participants, the data from this study can only be shared with subsequent agreement from research participants and after complying with a series of conditions. Please communicate directly with the first author regarding access to data.

\section{Competing interests}

The authors declare they have no competing interests.

\section{Funding}

The researchers did not apply for or receive any funding for this research.

\section{Acknowledgements}

Not applicable

\section{Authors' contributions}

LI designed the study and conducted the literature review. GC conducted the policy review. LI and GC conducted primary data collection through interviews. LI analysed the data and wrote the manuscript. GC and CV critically reviewed the manuscript. All authors read and approved the final manuscript.

\section{References}

1. Renfrew MJ, Cheyne H, Craig J, Duff E, Dykes F, Hunter B, Lavender T, Page L, Ross-Davie M, Spiby H et al: Sustaining quality midwifery care in a pandemic and beyond. Midwifery 2020, 88:102759.

2. Coxon K, Turienzo CF, Kweekel L, Goodarzi B, Brigante L, Simon A, Lanau MM: The impact of the coronavirus (COVID-19) pandemic on maternity care in Europe. Midwifery 2020, 88:102779.

3. Gutschow K, Davis-Floyd R: The Impacts of COVID-19 on US Maternity Care Practices: A Followup Study. Front Sociol 2021, 6:655401.

4. Sanders J, Blaylock R: "Anxious and traumatised": Users' experiences of maternity care in the UK during the COVID-19 pandemic. Midwifery 2021, 102:103069.

5. Wilson AN, Sweet L, Vasilevski V, Hauck Y, Wynter K, Kuliukas L, Szabo RA, Homer CSE, Bradfield Z: Australian women's experiences of receiving maternity care during the COVID-19 pandemic: A crosssectional national survey. Birth 2021, n/a(n/a).

6. Kotlar B, Gerson E, Petrillo S, Langer A, Tiemeier H: The impact of the COVID-19 pandemic on maternal and perinatal health: a scoping review. Reproductive Health 2021, 18(1):10.

7. DeBolt CA, Bianco A, Limaye MA, Silverstein J, Penfield CA, Roman AS, Rosenberg HM, Ferrara L, Lambert C, Khoury R et al: Pregnant women with severe or critical coronavirus disease 2019 have increased composite morbidity compared with nonpregnant matched controls. Am J Obstet Gynecol 2021, 224(5):510.e511-510.e512. 
8. Zambrano LD, Ellington S, Strid P, Galang RR, Oduyebo T, Tong VT, Woodworth KR, Nahabedian JF, 3rd, Azziz-Baumgartner E, Gilboa SM et al: Update: Characteristics of Symptomatic Women of Reproductive Age with Laboratory-Confirmed SARS-CoV-2 Infection by Pregnancy Status - United States, January 22-October 3, 2020. MMWR Morb Mortal Wkly Rep 2020, 69(44):1641-1647.

9. NHS encourages pregnant women to get COVID-19 vaccine [https://www.england.nhs.uk/2021/10/nhs-encourages-pregnant-women-to-get-covid-19-vaccine/]

10. American Society for Reproductive Medicine: Patient Management and Clinical Recommendations during the Coronavirus (Covid-19) Pandemic - Vaccination: Combating Hesitency and Misinformation. In.: American Society for Reproductive Medicine; 2021.

11. Royal College of Obstetricians \& Gynaecologists: The RCOG and the RCM respond to misinformation around Covid-19 vaccine and fertility. In.; 2021.

12. UK Health Security Agency: COVID-19 vaccine surveillance report: Week 47. In.; 2021.

13. Shimabukuro TT, Kim SY, Myers TR, Moro PL, Oduyebo T, Panagiotakopoulos L, Marquez PL, Olson CK, Liu R, Chang KT et al: Preliminary Findings of mRNA Covid-19 Vaccine Safety in Pregnant Persons. New England Journal of Medicine 2021, 384(24):2273-2282.

14. Jardine J, Relph S, Magee L, von Dadelszen P, Morris E, Ross-Davie M, Draycott T, Khalil A: Maternity services in the UK during the coronavirus disease 2019 pandemic: a national survey of modifications to standard care. BJOG: An International Journal of Obstetrics \& Gynaecology 2021, 128(5):880889.

15. Sanders JB, R.: Users' experiences of COVID-19 maternity service changes. In: WRISK Study. 2020.

16. Coxon $\mathrm{K}$, Chisholm A, Malouf R, Rowe R, Hollowell J: What influences birth place preferences, choices and decision-making amongst healthy women with straightforward pregnancies in the UK? A qualitative evidence synthesis using a 'best fit' framework approach. BMC Pregnancy and Childbirth 2017, 17(1):103.

17. Betteley C: Covid: Pregnant women 'opt for C-section' to ensure partners at birth. In: BBC News. bbc.co.uk: BBC; 2020.

18. Romanis EC, Nelson A: Homebirthing in the United Kingdom during COVID-19. Medical Law International 2020, 20(3):183-200.

19. Greenfield M, Payne-Gifford S, McKenzie G: Between a Rock and a Hard Place: Considering “Freebirth" During Covid-19. Frontiers in Global Women's Health 2021, 2(5).

20. Preis H, Mahaffey B, Heiselman C, Lobel M: Pandemic-related pregnancy stress and anxiety among women pregnant during the coronavirus disease 2019 pandemic. Am J Obstet Gynecol MFM 2020, 2(3):100155.

21. Gildner TE, Thayer ZM: Birth plan alterations among American women in response to COVID-19. Health Expectations 2020, 23(4):969-971.

22. Tricco AC, Langlois, E. V. \& Straus, S. E.: Rapid Reviews to Strengthen Health Policy and Systems: A Practical Guide. In. Geneva: World Health Organization; 2017. 
23. Gale NK, Heath G, Cameron E, Rashid S, Redwood S: Using the framework method for the analysis of qualitative data in multi-disciplinary health research. BMC Medical Research Methodology 2013, 13(1):117.

24. Vindrola-Padros C, Johnson GA: Rapid Techniques in Qualitative Research: A Critical Review of the Literature. Qualitative Health Research 2020, 30(10):1596-1604.

25. Office for National Statistics: Provisional births in England and Wales: 2020. In., 7 December 2020 edn. ons.gov.uk: Office for National Statistics; 2020.

26. Bohren MA, Hofmeyr GJ, Sakala C, Fukuzawa RK, Cuthbert A: Continuous support for women during childbirth. Cochrane Database of Systematic Reviews 2017(7).

27. Symon AG, Dugard P, Butchart M, Carr V, Paul J: Care and environment in midwife-led and obstetricled units: A comparison of mothers' and birth partners' perceptions. Midwifery 2011, 27(6):880-886.

28. Almeida M, Shrestha AD, Stojanac D, Miller LJ: The impact of the COVID-19 pandemic on women's mental health. Archives of Women's Mental Health 2020, 23(6):741-748.

29. Birthplace Collaborative Group: Perinatal and maternal outcomes by planned place of birth for healthy women with low risk pregnancies: the Birthplace in England national prospective cohort study. BMJ 2011, 343:d7400.

30. Reitsma A, Simioni J, Brunton G, Kaufman K, Hutton EK: Maternal outcomes and birth interventions among women who begin labour intending to give birth at home compared to women of low obstetrical risk who intend to give birth in hospital: A systematic review and meta-analyses. EClinicalMedicine 2020, 21.

31. Handelzalts JE, Zacks A, Levy S: The association of birth model with resilience variables and birth experience: Home versus hospital birth. Midwifery 2016, 36:80-85.

32. Jouhki MR, Suominen T, Åstedt-Kurki P: Giving birth on our own terms-Women's experience of childbirth at home. Midwifery 2017, 53:35-41.

33. Lindgren $\mathrm{H}$, Erlandsson $\mathrm{K}$ : Women's Experiences of Empowerment in a Planned Home Birth: A Swedish Population-based Study. Birth 2010, 37(4):309-317.

34. The Kings Fund/Ipsos MORI: The public and the NHS: What's the deal? 2018.

35. NHS England: Supporting pregnant women using maternity services during the coronavirus pandemic: Actions for NHS providers. In.: NHS England; 2020.

36. Liao SC, Chang YS, Chien LY: The maternity response to COVID-19: An example from one maternity unit in Taiwan. Midwifery 2020, 88:102756.

\section{Tables}

Table 1 is available in the Supplemental Files section. 
Table 2

Place of birth and mode of delivery

\begin{tabular}{|lllll|}
\hline $\begin{array}{l}\text { Place of } \\
\text { birth }\end{array}$ & OU & Birth centre & Homebirth & $\begin{array}{l}\text { Attempted homebirth, } \\
\text { transferred to OU }\end{array}$ \\
\hline $\begin{array}{l}\text { Mode of } \\
\text { delivery }\end{array}$ & $\begin{array}{l}\text { Vaginal delivery, } \\
\text { unassisted }\end{array}$ & $\begin{array}{l}\text { Vaginal delivery, } \\
\text { assisted }\end{array}$ & $\begin{array}{l}\text { C-section, } \\
\text { elective }\end{array}$ & C-section, emergency \\
\hline 26 & 13 & 3 & 4 \\
\hline
\end{tabular}

\section{Supplementary Files}

This is a list of supplementary files associated with this preprint. Click to download.

- Table1.jpg

- Additionalfile.docx 